\title{
Utility of Peripheral Film Findings and its Correlation with Automated Analyzer - An Audit from Tertiary Care Hospital
}

\author{
Sidra Asad, Imran Ahmed, Natasha Ali ${ }^{1}$
}

Departments of Pathology and Laboratory Medicine and ${ }^{1}$ Pathology and Laboratory Medicine and Oncology, The Aga Khan University, Karachi, Pakistan

Address for correspondence: Dr. Natasha Ali, E-mail: natasha.ali@aku.edu

\section{ABSTRACT}

Background and Objective: With the advent of automated hematology analyzer, the use of traditional microscopy of blood film has become limited. The objective of our study was to determine the percentage of peripheral blood smear review in our institution in the era of automation and to identify reasons of manual review.

Materials and Methods: This was a prospective audit from January 1, 2015, to January 15, 2015. Consecutive complete blood count $(\mathrm{CBC})$ samples and peripheral smear requests made up the sample size. All age groups and genders were included. CBCs were performed on Sysmex XE-5000. The variables to be analyzed included inpatient and outpatient samples, frequency of peripheral film review, identifying reasons of smear review, and addition of information missed by the automated analyzer.

Results: We analyzed 1200 consecutive CBC samples. Peripheral smear was reviewed in 500 (42\%) of the cases of which, 241 were inpatient, and 259 were outpatient samples. In 384/500, the findings of hematology analyzer correlated with peripheral smear review. Flags identified included nucleated red blood cells (NRBCs) in 155 (40\%), immature white blood cell (WBC) 129 (34\%), and atypical lymphocytes 100 (26\%). In 23\% of the cases, the analyzer missed important findings. The sensitivity of abnormal histogram in our study was $91.3 \%$, while the sensitivity of abnormal parameters was $100 \%$.

Conclusion: Peripheral smear review was performed in $42 \%$ of the cases. The analyzer identified NRBC, immature WBC precursors, and atypical lymphocytes as the most common abnormality. The information correlated in $77 \%$ of the cases.

Key words: Automated analyzer, complete blood count, peripheral film

\section{INTRODUCTION}

xamination of properly prepared peripheral blood film offers invaluable information about morphological changes which are not provided by automated instruments. It also provides quality assurance information of complete blood counts (CBCs) data generated by laboratory hematology analyzers.

It is a useful and economical diagnostic tool which can be used both in adults and children and despite the advent of automated blood cell analyzers,

\begin{tabular}{|l|l|}
\hline \multicolumn{2}{|c|}{ Access this article online } \\
\hline Quick Response Code: & Website: \\
& \\
\hline
\end{tabular}

examination of peripheral smear by the experienced technologist, and qualified hematologists cannot be repudiated. Rapid, reliable access to information about a variety of hematologic disorders is provided; in some cases, review of peripheral smear along with clinical data may be sufficient enough to establish a diagnosis. ${ }^{[1]}$

The blood film reflects functional status of the bone marrow, the factory producing all blood elements and its examination is particularly

This is an open access article distributed under the terms of the Creative Commons Attribution-NonCommercial-ShareAlike 3.0 License, which allows others to remix, tweak, and build upon the work non-commercially, as long as the author is credited and the new creations are licensed under the identical terms.

For reprints contact: reprints@medknow.com

How to cite this article: Asad S, Ahmed I, Ali N. Utility of peripheral film findings and its correlation with automated analyzer - an audit from tertiary care hospital. J Lab Physicians 2017;9:1-4. 
important when assessing patients with cytopenias. Other conditions in which the peripheral film findings can be diagnostic include microangiopathic hemolytic anemia, hemoglobinopathy, myeloproliferative disorders, and parasitic infections (especially malaria).

With the advent hematology analyzers, microscopic review of peripheral blood film is declining. Sophistication of analyzers has increased to the point that they are able to provide cells counts, differentials, plots, and histograms.

Microscopy and manual differential counting, in most of the automated laboratories, is restricted to cases in which the instrument "flags" the potential presence of abnormal cells or in cases where findings may interfere with analysis (such as overlap in the distribution of different cell types or interference from matrix components). In cases of clinical suspicion of leukemia, review of the peripheral blood smear is mandatory to make the presumptive diagnosis. There are other morphological findings also which may have a clinical significance which cannot be reliably identified by the various automated analyzers. These other findings include the presence of giant platelets, platelet clumps, basophilic stippling, hypersegmented neutrophils, red cell fragments, and Howell-Jolly bodies. ${ }^{[2]}$

Since the inception of automated differential counting methods, manual blood smear review is recommended as a validation, rather than as a replacement of automated methods. ${ }^{[3]}$

Reviewing peripheral smear and performing manual differential counts need the expertise of well-trained laboratory staff and leads to under productivity and consumption of time. This has a much greater impact when the automated and manual results are similar leading to decreased working capacity of house staff. In this era of medical advancements and automation, it is important to reduce the workload and improve turnaround time to combat the continuing pressure on laboratory resources. ${ }^{[4]}$ However for that purpose, important diagnostic information must not be missed by completely relying on morphological findings given by the analyzer as automation does not provide all the information that is potentially important to the physician. ${ }^{[5]}$ The Colleges of American Pathologists (CAP) have conducted numerous studies though Q-probes program to determine performance benchmarks in Pathology. Novis et al. in 2006 have reported peripheral smear review frequency of $26.7 \%{ }^{\left[{ }^{[6]}\right.}$

With this background, our objective was to determine the frequency of peripheral smear review in our institution and compare it with CAP standards. We also wanted to correlate the findings of peripheral smear and analyzer flags and to identify information which was missed by the analyzer.

\section{MATERIALS AND METHODS}

This was a retrospective audit conducted in the section of Haematology, Department of Pathology and Laboratory Medicine of The Aga Khan University (AKU) located in Southern Pakistan. AKU is a tertiary care hospital with well-equipped Clinical Laboratory offering over 700 test menu in different sections and is considered as a national reference setup. In our section, approximately 1500 CBCs are reported every day. Peripheral blood film is made of only those blood samples which trigger laboratory policy of smear review defined as abnormal counts (set of criteria established by our expert opinion consensus) and flagging given by the analyzer.

For the study, data for 2 weeks (January 1, 2015-January 15, 2015) were collected from three traditional shifts with simple random sampling technique as the sampling frame. All age groups and genders were included. Specimens with clots, obvious hemolysis, insufficient amount of sample, incorrect addressograph, or wrong vacutainer were excluded.

Automated CBCs were performed on Sysmex XE-5000 hematology analyzers. Inpatient samples were received from all hospital locations (i.e., emergency department, wards, special care units, etc.). Outpatient stations included clinics and outside referrals by physicians.

Peripheral films were prepared by Sysmex SP-1000i automated hematology slide preparation unit. Each "positive smear" was reviewed by an experienced technologist and verified by a hematology resident/consultant. For each specimen on which a manual review was performed, we documented the hemoglobin value, white blood cell (WBC) count, and platelet count the primary reason for the review. The frequency of manual peripheral blood smear review, the manual scan review, and the manual differential count was also determined. Data were collected on a predesigned questionnaire and IBM SPSS Statistics for Windows, Version 19.0. Armonk, NY: IBM Corp., was used for data entry and analysis.

\section{RESULTS}

During the review period, we analyzed 1200 consecutive CBC samples. Peripheral smear was reviewed in $n=500(42 \%)$ of the cases using random sampling technique. Of these 
500 samples, 241 were inpatient and 259 were outpatient samples. In 384/500, the findings of hematology analyzer correlated with peripheral smear review. Flags which were identified included nucleated red blood cells in 155 (40\%), immature WBC 129 (34\%), and atypical lymphocytes $100(26 \%)$. In $23 \%$ (116) of the cases, the analyzer missed important findings. These included abnormalities in hemoglobin indices in 54 (47\%), WBCs differential counts in $36(31 \%)$ and large platelets in $26(22 \%)$. Furthermore, the sensitivity of abnormal histogram in our study was $91.3 \%$, while the specificity of the same was $8.17 \%$. Accordingly, the sensitivity of abnormal parameters was $100 \%$, and the specificity of the same was $0 \%$. Further details are given in Table 1.

\section{DISCUSSION}

Microscopic examination and morphological assessment are an essential part of $\mathrm{CBC}$ reporting that provides crucial information apart from the cell counts. Review of peripheral blood smear serves to ensure that no clinically significant finding is missed, besides providing a clue to the diagnosis, when interpreted by a physician. ${ }^{[7]}$ This information is now provided by automated analyzers with more sensitivity reducing the need of manual examination. The purpose of automation is to provide faster reportable results, to reduce the technologist hands-on time, in addition to providing high quality and precision. ${ }^{[8]}$

Defining acceptable and safe rates for microscopic examination of the blood smear is crucial to ensure the quality of the results, but reported rates are highly variable. ${ }^{[0]}$

Comar et al. verified the review criteria for automated blood counts suggested by the International Society for Laboratory Haematology and their results showed microscopic review rate of $46.03 \%$ with false negatives of $6.73 \%$, false positives of $23.27 \%$, and efficiency equivalent to $70.0 \%$. After adapting the review criteria, the microscopic review rate dropped to $37.3 \%$ with false negatives reaching $15.5 \%$, false positives of $10.5 \%$, and efficiency of $73.8 \%{ }^{[10]}$

Pratumvinit and his colleagues evaluated their criteria for manual smear review and after optimization, their review rate was found to be $24.2 \%$ with an efficiency of $87.13 \%$ and false negative rate of $2.98 \%{ }^{[11]}$

Another study by Xing et al. established review criteria for their analyzer. After modifying the criteria, the review rate was $34.2 \%$ versus $50.2 \%$, false negative rate was $5.5 \%$ versus $4.2 \%$, and false positive rate was $28.1 \%$ versus $18.7 \%$. ${ }^{[12]}$ In our study, the sensitivity of abnormal histogram was $91.3 \%$, abnormal parameter was $100 \%$, and the sensitivity for flagging was $97.9 \%$. Our manual smear review rate was $42 \%$, which is approximately twice as compared to CAP standards. Since we used random sampling technique, the possibility of missing important diagnostic findings cannot be entirely excluded which is the limitation of the study.

The clinical laboratory of $\mathrm{AKU}$ is a reference laboratory where $\mathrm{CBC}$ s are received not only from admitted patients but also from collection points located all over the country and outside Pakistan. Our goal of performing this audit was to reduce the workload of microscopy as far as possible to increase laboratory efficiency in terms of time, workforce, and resources by relying on automated analyzer but without missing vital information, i.e., false negative results. Our laboratory is in the process of obtaining CAP accreditation. The processes and standard operating procedures have been modified accordingly. With the accreditation underway and based on the results of this audit, our way forward will be to redefine our triggers for peripheral smear review which will not only decrease workload in laboratory but also shorten the turnaround time of $\mathrm{CBC}$ reporting. Once the changes have been implemented, we will perform a re-audit to complete the cycle.

\section{CONCLUSION}

It is concluded that the frequency of peripheral blood smear review in our setup was $42 \%$ with our less stringent criteria. In $77 \%$ of cases, the findings of peripheral blood smears review correlated with that of the analyzer.

\section{Ethical approval}

The study was given exemption from ethical approval by the Ethical Review Committee of The Aga Khan University (3573-Pat-ERC-15).

\begin{tabular}{lccccccc}
\hline \multicolumn{2}{l}{ Table 1: Diagnostic accuracy } & & & & \\
\hline Variable & $\begin{array}{c}\text { Sensitivity } \\
(\%)\end{array}$ & $\begin{array}{c}\text { Specificity } \\
(\%)\end{array}$ & $\begin{array}{c}\text { Positive predictive } \\
\text { value (\%) }\end{array}$ & $\begin{array}{c}\text { Negative predictive } \\
\text { value (\%) }\end{array}$ & $\begin{array}{c}\text { Positive } \\
\text { likelihood ratio }\end{array}$ & $\begin{array}{c}\text { Negative } \\
\text { likelihood ratio }\end{array}$ & $\begin{array}{c}\text { Accuracy } \\
(\%)\end{array}$ \\
\hline Abnormal histogram & 91.3 & 8.17 & 91.3 & 8.17 & 0.99 & -1.06 \\
Abnormal parameter & 100 & 0 & - & - & - & 48.6 \\
Flag & 97.9 & 4.2 & 95.5 & 4.2 & 1.01 & - \\
\hline
\end{tabular}




\section{Financial support and sponsorship}

Nil.

\section{Conflicts of interest}

There are no conflicts of interest.

\section{REFERENCES}

1. Adewoyin AS, Nwogoh B. Peripheral blood film - A review. Ann Ib Postgrad Med 2014;12:71-9.

2. Bain BJ. Diagnosis from the blood smear. N Engl J Med 2005;353:498-507.

3. Lantis KL, Harris RJ, Davis G, Renner N, Finn WG. Elimination of instrument-driven reflex manual differential leukocyte counts. Optimization of manual blood smear review criteria in a high-volume automated hematology laboratory. Am J Clin Pathol 2003;119:656-62.

4. Briggs CJ, Linssen J, Longair I, Machin SJ. Improved flagging rates on the Sysmex XE-5000 compared with the XE-2100 reduce the number of manual film reviews and increase laboratory productivity. Am J Clin Pathol 2011;136:309-16.
5. Ford J. Red blood cell morphology. Int J Lab Hematol 2013;35:351-7.

6. Novis DA, Walsh M, Wilkinson D, St. Louis M, Ben-Ezra J. Laboratory productivity and the rate of manual peripheral blood smear review: A College of American Pathologists Q-Probes study of 95,141 complete blood count determinations performed in 263 institutions. Arch Pathol Lab Med 2006;130:596-601.

7. Gulati G, Song J, Florea AD, Gong J. Purpose and criteria for blood smear scan, blood smear examination, and blood smear review. Ann Lab Med 2013;33:1-7.

8. Grotto HZ. Why and how validate criteria by manual smear review to improve laboratory productivity? Rev Bras Hematol Hemoter 2015;37:67-8.

9. Froom P, Havis R, Barak M. The rate of manual peripheral blood smear reviews in outpatients. Clin Chem Lab Med 2009;47:1401-5.

10. Comar SR, Malvezzi M, Pasquini R. Are the review criteria for automated complete blood counts of the International Society of Laboratory Hematology suitable for all hematology laboratories? Rev Bras Hematol Hemoter 2014;36:219-25.

11. Pratumvinit B, Wongkrajang P, Reesukumal K, Klinbua C, Niamjoy P. Validation and optimization of criteria for manual smear review following automated blood cell analysis in a large university hospital. Arch Pathol Lab Med 2013;137:408-14.

12. Xing Y, Wang JZ, Pu CW, Shang K, Yan ZL, Bai YZ, et al. Establishment and evaluation of review criteria for ADVIA 120/2120 and different series of hematology analyzers. Zhonghua Yi Xue Za Zhi 2010;90:1526-30. 